\title{
Asymptotic behavior for the semilinear reaction-diffusion equations with memory
}

\author{
Jiangwei Zhang ${ }^{1}$, Yongqin Xie ${ }^{1 *}$ (D), Qingqing Luo ${ }^{1}$ and Zhipiao Tang ${ }^{1}$
}

\author{
"Correspondence: \\ xieyqmath@126.com \\ 1 School of Mathematics and and \\ Statistics, Changsha University of \\ Science and Technology, Changsha, \\ China
}

\begin{abstract}
In this paper, we consider the dynamics of a reaction-diffusion equation with fading memory and nonlinearity satisfying arbitrary polynomial growth condition. Firstly, we prove a criterion in a general setting as an alternative method (or technique) to the existence of the bi-spaces attractors for the nonlinear evolutionary equations (see Theorem 2.14). Secondly, we prove the asymptotic compactness of the semigroup on $L^{2}(\Omega) \times L_{\mu}^{2}\left(\mathbb{R} ; H_{0}^{1}(\Omega)\right)$ by using the contractive function, and the global attractor is confirmed. Finally, the bi-spaces global attractor is obtained by verifying the asymptotic compactness of the semigroup on $L^{p}(\Omega) \times L_{\mu}^{2}\left(\mathbb{R} ; H_{0}^{\prime}(\Omega)\right)$ with initial data in $L^{2}(\Omega) \times L_{\mu}^{2}\left(\mathbb{R} ; H_{0}^{1}(\Omega)\right)$.
\end{abstract}

Keywords: Reaction-diffusion equation; Memory; The bi-spaces asymptotic compact; Global attractor; Arbitrary polynomial growth

\section{Introduction}

The aim of this paper is to analyze the long-time behavior of solutions of the following semilinear reaction-diffusion equations with fading memory:

$$
u_{t}-\Delta u-\int_{0}^{\infty} k(s) \Delta u(t-s) d s+f(u)=g
$$

with Dirichlet boundary condition, where $g \in L^{2}(\Omega), \Omega \subset \mathbb{R}^{n}(n \geq 3)$ is a bounded domain with smooth boundary, nonlinearity $f$, and the memory kernel function $k(s)$ satisfying posterior adaptation hypothesis which will be given below (see, e.g., [3, 4]).

This kind of integro-differential reaction-diffusion equation is well known and can be interpreted, for instance, as a model of heat diffusion with memory which also accounts for a reaction process depending on the temperature itself (see, e.g., $[1,8,10,11,15,16]$ and the references therein). This equation also appears as the model of polymers and high viscosity liquids, the diffusion process is influenced by the past historical growth, which is mainly represented in the convolution term of fading memory characterizing diffusion species to a suitable memory core [12]. The energy dissipative speed for equation (1.1) is faster than the common reaction diffusion equation $(k \equiv 0)$. The heat energy transmission is not only affected by the present external force but also by the historical external force, and as time elapses, this effect will gradually decline.

As $k=0$, equation (1.1) reduces to usual reaction diffusion, the long-time behavior and related issues of solutions of this problem have been extensively discussed in recent years

(c) The Author(s) 2019. This article is distributed under the terms of the Creative Commons Attribution 4.0 International License (http://creativecommons.org/licenses/by/4.0/), which permits unrestricted use, distribution, and reproduction in any medium, provided you give appropriate credit to the original author(s) and the source, provide a link to the Creative Commons license, and indicate if changes were made. 
(see, e.g., $[2,5,14,17,19-23,26]$ and the references therein). The above problem has also been analyzed within the theory of infinite-dimensional dynamical systems $[6,8,10]$, following Dafermos' idea of introducing an additional variable $\eta^{t}$, the past history of $u$, whose evolution is ruled by a first-order hyperbolic equation (see, e.g., $[7,8]$ and the references therein). Thus the original problem (1.1) can be transformed into a dynamical system on a phase space with two components (1.13) or (1.15) (see, e.g., [3, 4]). In [9], the existence of an absorbing set for heat conduction equation with memory has been proved in $L^{2}(\Omega) \times L_{\mu}^{2}\left(\mathbb{R} ; H_{0}^{1}(\Omega)\right)$ and $H_{0}^{1}(\Omega) \times L_{\mu}^{2}\left(\mathbb{R} ; H_{0}^{1}(\Omega) \cap H^{2}(\Omega)\right)$. Next, in [8], when the nonlinear term $f$ satisfies critical growth, the existence of global attractor is obtained in $L^{2}(\Omega) \times L_{\mu}^{2}\left(\mathbb{R} ; H_{0}^{1}(\Omega)\right)$. In [10], when nonlinearity is subcritical, the author obtains the existence of bounded absorbing sets in $H_{0}^{1}(\Omega) \times L_{\mu}^{2}\left(\mathbb{R} ; H^{2}(\Omega) \cap H_{0}^{1}(\Omega)\right)$. In [25], the bispaces global attractor $\mathscr{A}$ is confirmed in $\left(L^{2}(\Omega) \times L_{\mu}^{2}\left(\mathbb{R} ; H_{0}^{1}(\Omega)\right), H_{0}^{1}(\Omega) \times L_{\mu}^{2}\left(\mathbb{R} ; H_{0}^{1}(\Omega)\right)\right.$ ), the nonlinearity $f$ satisfies the polynomial growth of arbitrary order and bi-spaces global attractor $\mathscr{A} \subset H_{0}^{1}(\Omega) \cap H^{2}(\Omega) \times L_{\mu}^{2}\left(\mathbb{R} ; H_{0}^{1}(\Omega) \cap H^{2}(\Omega)\right)$.

As we know, if we want to prove the existence of global attractors, the key point is to obtain the compactness of the semigroup in some sense. Noticing that the nonlinearity satisfies arbitrary polynomial growth condition and Eq. (1.1) has no higher regularity owing to fading memory and the embedding $L_{\mu}^{2}\left(\mathbb{R} ; H^{2}(\Omega) \cap H_{0}^{1}(\Omega)\right) \hookrightarrow L_{\mu}^{2}\left(\mathbb{R} ; H_{0}^{1}(\Omega)\right)$ is noncompact, so Sobolev embeddings are no longer compact and the asymptotic compactness of solutions cannot be obtained by the usual method. To overcome the difficulty of the noncompact embedding, in [18], using the idea of the contractive function method, the authors consider the asymptotic behavior of nonautonomous wave equations, they prove that the family of processes is uniformly asymptotically compact under the nonlinearity $f$ satisfying critical growth (see, e.g., [18]). In [23, 24], the authors consider the asymptotic behavior of nonclassical diffusion equations with the nonlinearity satisfying arbitrary polynomial growth by the (uniform) asymptotic contractive function method, but the initial data $z_{0}$ and the solution $z$ of Eq. (1.1) belong to the same space $\left.z_{0}, z(t) \in H_{0}^{1}(\Omega)\right) \times L_{\mu}^{2}\left(\mathbb{R} ; H_{0}^{1}(\Omega)\right)$. Unfortunately, it is well known that we cannot get that the semigroup $\{S(t)\}_{t \geq 0}$ is the contractive semigroup on $L^{p}(\Omega) \times L_{\mu}^{2}\left(\mathbb{R} ; H_{0}^{1}(\Omega)\right)$ directly when the nonlinearity $f$ satisfies the polynomial growth of arbitrary order.

In this paper, we take the extension of the method in $[18,23,24,27,28]$ to the normto-weak continuous semigroup on a product space. The abstract result, which proves the existence of bi-space global attractors, is obtained (see Theorem 2.9). As an application, we confirm the existence of $\left(L^{2}(\Omega) \times L_{\mu}^{2}\left(\mathbb{R}^{+}, H_{0}^{1}(\Omega)\right), L^{p}(\Omega) \times L_{\mu}^{2}\left(\mathbb{R}^{+}, H_{0}^{1}(\Omega)\right)\right)$-global attractor $\mathscr{A}$ for the reaction-diffusion equation with memory and arbitrarily growing nonlinearity.

The nonlinearity $f$ satisfies $f \in C^{1}, f(0)=0$, and the arbitrary order exponential growth condition

$$
\alpha_{1}|s|^{p}-\beta_{1} \leq f(s) s \leq \alpha_{2}|s|^{p}+\beta_{2}, \quad \forall s \in \mathbb{R}, p \geq 2,
$$

where $\alpha_{i}, \beta_{i}(i=1,2)$, and $l$ are positive constants and dissipative conditions

$$
f^{\prime}(s) \geq-l, \quad \forall s \in \mathbb{R}
$$

Let $F(s)=\int_{0}^{s} f(\tau) d \tau$, by $(1.2)$ there exist constants $\tilde{\alpha}_{i}, \tilde{\beta}_{i}>0(i=1,2)$ such that

$$
\tilde{\alpha}_{1}|s|^{p}-\tilde{\beta}_{1} \leq F(s) \leq \tilde{\alpha}_{2}|s|^{p}+\tilde{\beta}_{2} .
$$


Concerning the memory kernel function $k(s)$, let $\mu(s)=-k^{\prime}(s)$. We hypothesize

$$
\mu \in C^{1}\left(\mathbb{R}^{+}\right) \cap L^{1}\left(\mathbb{R}^{+}\right), \quad \mu(s) \geq 0, \quad \mu^{\prime}(s) \leq 0, \quad \forall s \in \mathbb{R}^{+},
$$

and there are constants $\delta, \gamma>0$ such that

$$
\mu^{\prime}(s)+\delta \mu(s) \leq 0, \quad \forall s \in \mathbb{R}^{+} .
$$

From (1.5) and (1.6), we get

$$
\mu(\infty)=\lim _{s \rightarrow \infty} \mu(s)=0
$$

and

$$
m_{0}=\int_{0}^{\infty} \mu(s) d s
$$

Next, we introduce the past history of $u$, that is,

$$
\eta^{t}=\eta^{t}(x, s):=\int_{0}^{s} u(x, t-\tau) d \tau, \quad s \in \mathbb{R}^{+}
$$

then $\eta_{t}^{t}=\frac{\partial}{\partial t} \eta^{t}, \eta_{s}^{t}=\frac{\partial}{\partial s} \eta^{t}$, and

$$
\eta_{t}^{t}=-\eta_{s}^{t}+u
$$

Historical variable $u_{0}(\cdot,-s)$ of $u$ satisfies the following conditions: there exist positive constant $\Re$ and $\sigma \leq \delta$ ( $\delta$ is from (1.6)) such that

$$
\int_{0}^{\infty} e^{-\sigma s}\left\|u_{0}(-s)\right\|_{0}^{2} d s \leq \Re
$$

Combining with (1.7) and (1.9), properties of Lebesgue integral and partial integral, we get

$$
\int_{0}^{\infty} k(s) \Delta u(t-s) d s=\int_{0}^{\infty} \mu(s) \Delta \eta^{t}(s) d s
$$

Hence, (1.1) is transformed into the following system:

$$
\left\{\begin{array}{l}
u_{t}-\Delta u-\int_{0}^{\infty} \mu(s) \Delta \eta^{t}(s) d s+f(u)=g \\
\eta_{t}^{t}=-\eta_{s}^{t}+u
\end{array}\right.
$$

with initial-boundary conditions

$$
\left\{\begin{array}{l}
\left.u(x, t)\right|_{\partial \Omega}=0,\left.\quad \eta^{t}(x, s)\right|_{\partial \Omega \times \mathbb{R}^{+}}=0, \quad t \geq 0, \\
u(x, 0)=u_{0}(x), \quad \eta^{0}(x, s)=\int_{0}^{s} u_{0}(x,-\tau) d \tau, \quad(x, s) \in \Omega \times \mathbb{R}^{+} .
\end{array}\right.
$$


Now, denoting $z(t)=\left(u(t), \eta^{t}\right)$ as the solution of problem (1.13) with initial data $z_{0}=$ $\left(u_{0}, \eta^{0}\right)$ and setting

$$
\mathcal{Q} z=\left(\Delta u+\int_{0}^{\infty} \mu(s) \Delta \eta^{t}(s) d s, u-\eta_{s}^{t}\right)
$$

and

$$
\mathcal{G}(z)=(g-f, 0)
$$

problem (1.13) with initial-boundary conditions (1.14) assumes the compact form

$$
\begin{cases}z_{t}=\mathcal{Q} z+\mathcal{G}(z) & \text { in } \Omega \times \mathbb{R}^{+}, \\ z(x, 0)=z_{0} & \text { in } \Omega, \\ z(x, t)=0 & \text { on } \partial \Omega \times \mathbb{R}^{+} .\end{cases}
$$

For convenience, hereafter the $m(e)$ or $|e|$ denote the Lebesgue measure of $e \subset \mathbb{R}^{n}, C$ is an arbitrarily positive constant, which may be different from line to line, even in the same line. Let $|u|$ be the modular (or absolute value) of $u,|\cdot|_{p}$ be the norm of $L^{p}(\Omega)(p \geq 1)$ (particular denoting $H=L^{2}(\Omega)$ ) and $\|\cdot\|_{0}=|\nabla \cdot|_{2}$ be the equivalent norm of $V=H_{0}^{1}(\Omega)$. Denote $A=-\Delta, D(A)=H_{0}^{1}(\Omega) \cap H^{2}(\Omega)$ with the equivalent norm of $\|\cdot\|_{1}=|\Delta \cdot|_{2}$. Let $X$ be a Banach space, $X^{*}$ be the dual space of $X$, and $L_{\mu}^{2}\left(\mathbb{R}^{+}, X\right)$ be Hilbert spaces of functions $\varphi: \mathbb{R} \rightarrow X$, endowed with the inner product and norm respectively:

$$
\begin{aligned}
& \langle\varphi, \psi\rangle_{\mu, X}=\int_{0}^{\infty} \mu(s)\langle\varphi(s), \psi(s)\rangle_{X} d s, \quad \forall \psi \in X^{*} . \\
& \|\varphi\|_{\mu, X}^{2}=\int_{0}^{\infty} \mu(s)\|\varphi(s)\|_{X}^{2} d s .
\end{aligned}
$$

In particular, $\|\varphi\|_{\mu, 0}^{2}$ as $X=V$ and $\|\varphi\|_{\mu, 1}^{2}$ as $X=D(A)$.

Lemma 1.1 ([24]) Let $X$ be a Hilbert space, $I=[0, T], \forall T \geq 0$, memory kernel $\mu(s)$ satisfy (1.4) and (1.5). Then, for any $\eta^{t} \in C\left(I, L_{\mu}^{2}\left(\mathbb{R}^{+}, X\right)\right)$, the following estimate

$$
\left\langle\eta^{t}, \eta_{s}^{t}\right\rangle_{\mu, X} \geq \frac{\delta}{2}\left\|\eta^{t}\right\|_{\mu, X}^{2}
$$

holds, where $\delta$ is from (1.6).

Let $X, Y$ be two Banach spaces, we also define a class of Banach product spaces $\mathcal{M}=$ $X \times L_{\mu}^{2}\left(\mathbb{R}^{+}, Y\right)$ with norm $\|(\cdot, \cdot)\|_{\mathcal{M}}^{2}=\|\cdot\|_{X}^{2}+\|\cdot\|_{\mu, Y}^{2}$ In particular, $\mathcal{L}_{2}=H \times L_{\mu}^{2}\left(\mathbb{R}^{+}, V\right), \mathcal{L}_{p}=$ $L^{p}(\Omega) \times L_{\mu}^{2}\left(\mathbb{R}^{+}, V\right), \mathcal{M}_{1}=V \times L_{\mu}^{2}\left(\mathbb{R}^{+}, V\right)$, and $\mathcal{M}_{2}=D(A) \times L_{\mu}^{2}\left(\mathbb{R}^{+}, D(A)\right)$.

\section{Abstract results}

As mentioned earlier, our main work is to consider the existence of the $\left(\mathcal{L}_{2}, \mathcal{L}_{p}\right)$ )-global attractor for Eq. (1.1). Thus, we have to recall some basic concepts about the bi-space global attractor and then give a new criterion for the existence of bi-space attractors. 
Definition 2.1 ([26]) Let $X$ be a Banach space and $\{S(t)\}_{t \geq 0}$ be a family of operators on $X$. We say that $\{S(t)\}_{t \geq 0}$ is a norm-to-weak continuous semigroup on $X$ if $\{S(t)\}_{t \geq 0}$ satisfies:

(i) $S(0)=$ Id (the identity);

(ii) $S(t) S(s)=S(t+s) \forall t, s \geq 0$;

(iii) $S\left(t_{n}\right) x_{n} \rightarrow S(t) x$ if $t_{n} \rightarrow t$ and $x_{n} \rightarrow x$ in $X$.

In the evolution equation, this kind of semigroup corresponds to a solution that only satisfies weaker stability condition and, generally, it is neither continuous (i.e., norm-tonorm) nor weakly continuous (i.e., weak-to-weak). But obviously, the continuous semigroup and the weakly continuous semigroup are both norm-to-weak continuous semigroups. As is known to all, for some concrete problems, it is difficult to verify whether the semigroup is continuous or weakly continuous in a stronger normed space. However, it follows from the results described in what follows that the semigroup is norm-to-weak continuous in a stronger normed space.

Lemma $2.2([5,22,26])$ Let $X$ and $Y$ be two Banach spaces and $X^{*}, Y^{*}$ be their respective dual spaces, satisfying

$$
X \stackrel{i}{\hookrightarrow} Y \quad \text { and } \quad Y^{*} \stackrel{i^{*}}{\hookrightarrow} X^{*}
$$

where the injection $i: X \rightarrow Y$ is continuous and its adjoint $i^{*}: Y^{*} \rightarrow X^{*}$ is densely injective. Suppose also that $\{S(t)\}_{t \geq 0}$ is a semigroup on $X$ and $Y$. Assume furthermore that $\{S(t)\}_{t \geq 0}$ is a continuous semigroup or a weak continuous semigroup on $Y$. Then $\{S(t)\}_{t \geq 0}$ is a norm-toweak continuous semigroup on $X$ if and only if $\{S(t)\}_{t \geq 0}$ maps the compact subset of $X \times \mathbb{R}^{+}$ into the bounded set of $X$.

Definition 2.3 ([2,5]) A set $\mathscr{A} \subset X$, which is invariant, closed in $X$, compact in $Z$, and attracts bounded subsets of $X$ in the topology of $Z$, is called an $(X, Z)$-global attractor.

Definition 2.4 ([2, 5]) Let $\{S(t)\}_{t \geq 0}$ be a semigroup on a Banach space $X$. A set $B_{0} \subset Z$ is called $(X, Z)$-bounded absorbing set if, for any bounded subset $B$ of $X$, there is $T=T(B)$ such that $S(t) B \subset B_{0}$ provided that $t \geq T$.

Definition 2.5 ([2]) Let $\{S(t)\}_{t \geq 0}$ be a semigroup on a Banach space $X$. The semigroup $\{S(t)\}_{t \geq 0}$ is called $(X, Z)$-asymptotically compact if, for any bounded (in $\left.X\right)$ sequence $\left\{x_{n}\right\}_{n=1}^{\infty} \subset X$ and $t_{n} \geq 0, t_{n} \rightarrow \infty$ as $n \rightarrow \infty,\left\{S\left(t_{n}\right) x_{n}\right\}_{n=1}^{\infty}$ has a convergent subsequence with respect to the topology of $Z$.

Lemma 2.6 ([10]) Let $X$ and $Y$ be two Banach spaces with respective norms $\|\cdot\|_{X}$ and $\|\cdot\|_{Y}$, with $B \in \mathscr{B}(X) \cap \mathscr{B}(Y)$. Assume that $\left\{x_{n}\right\}_{n=1}^{\infty} \in B$ and $x_{n} \stackrel{\|\cdot\|_{X}}{\longrightarrow} x_{0}, x_{n} \stackrel{\|\cdot\|_{Y}}{\longrightarrow} y_{0}, x_{0}, y_{0} \in B$. Then

$$
x_{0}=y_{0},
$$

where $\mathscr{B}(X)$ denotes the collection of all bounded subsets of $X$. 
Definition 2.7 $([18,24])$ Let $X$ be a Banach space and B be a bounded subset of $X$. We call a function $\phi(\cdot, \cdot)$, defined on $X \times X$, a contractive function if, for any sequence $\left\{x_{n}\right\}_{n=1}^{\infty} \subset B$, there is a subsequence $\left\{x_{n_{k}}\right\}_{k=1}^{\infty} \subset\left\{x_{n}\right\}_{n=1}^{\infty}$ satisfying

$$
\lim _{k \rightarrow \infty} \lim _{l \rightarrow \infty} \phi\left(x_{n_{k}}, x_{n_{l}}\right)=0 .
$$

We denote the set of all contractive functions on $B \times B$ by $\mathfrak{E}(B)$.

In the following theorem, we present a new method (or technique) to verify the asymptotic compactness of a semigroup generated by evolutionary equations, which will be used in our later discussion.

Lemma 2.8 ([13, 18, 24] $)$ Let $X$ be a Banach space and B be a bounded subset of $X,\{S(t)\}_{t \geq 0}$ is a semigroup with a bounded absorbing set $B_{0}$ on $X$. Moreover, assume that, for any $\varepsilon>0$, there exist $T=T(B ; \varepsilon)$ and $\phi_{T}(\cdot, \cdot) \in \mathfrak{E}(B)$ such that

$$
\|S(T) x-S(T) y\|_{X} \leq \varepsilon+\phi_{T}(x, y), \quad \forall x, y \in B
$$

where $\phi_{T}$ depend on $T$. Then the semigroup $\{S(t)\}_{t \geq 0}$ is asymptotically compact in $X$.

Theorem 2.9 Let $X, Z$ be two Banach spaces satisfying

$$
Z \stackrel{i}{\hookrightarrow} X
$$

where the injection $i: Z \rightarrow X$ is continuous, and $\{S(t)\}_{t \geq 0}$ be a continuous semigroup on $X$ and a semigroup on $Z$. Suppose that $\{S(t)\}_{t \geq 0}$ has an $(X, X)$-global attractor. Then $\{S(t)\}_{t \geq 0}$ has an $(X, Z)$-global attractor provided that the following conditions hold:

(i) $\{S(t)\}_{t \geq 0}$ has an $(X, Z)$-bounded absorbing set $B_{0}$;

(ii) $\{S(t)\}_{t \geq 0}$ is $(X, Z)$-asymptotically compact.

Proof From the assumption that $\{S(t)\}_{t \geq 0}$ has an $(X, X)$-global attractor, we know that $\{S(t)\}_{t \geq 0}$ has an $(X, Z)$-bounded absorbing set. Because the embedding $Z \hookrightarrow X$ is continuous but not necessarily compact, $B_{0}$ is not only an $(X, Z)$-bounded absorbing set, but also an $(X, X)$-bounded absorbing set. Let

$$
\mathscr{A}=\bigcap_{s \geq 0} \widehat{\bigcup}_{t \geq s} S(t) B_{0}{ }^{2},
$$

here $\bar{A}^{Z}$ is the closure of $A$ in $Z$. Then, by the basic theory of dynamical systems (see [2, $17,19])$, it follows that $\mathscr{A}$ is a compact subset of $Z$ and, for any bounded $B \subset X$,

$$
\lim _{t \rightarrow \infty} \operatorname{dist}_{Z}(S(t) B, \mathscr{A})=0 .
$$

It is obvious that $\{S(t)\}_{t \geq 0}$ is a norm-to-weak continuous semigroup on $Z$. What remains is that we just need to prove that $\mathscr{A}$ is an invariant set of $\{S(t)\}_{t \geq 0}$ in $Z$, that is, for any $t \geq 0$,

$$
S(t) \mathscr{A}=\mathscr{A} .
$$


By the assumption that $\{S(t)\}_{t \geq 0}$ possesses an $(X, X)$-global attractor $\mathscr{B}$, hence $\mathscr{B}$ is an invariant set of $\{S(t)\}_{t \geq 0}$ in $X$. Now we prove that $\mathscr{A}=\mathscr{B}$.

From Lemma 2.2, $\{S(t)\}_{t \geq 0}$ is a norm-to-weak continuous semigroup on $Z$ and possesses a bounded absorbing set $B_{0}$. First, it is so easy to prove $\mathscr{B} \subset \mathscr{A}$. In fact, combining assumption (i) with definition (2.2) of $\mathscr{A}$, and $\mathscr{A}$ is an attracting set of $\{S(t)\}_{t \geq 0}$ in $X$. And yet $\mathscr{B}$ is the minimal attracting set, it follows that $\mathscr{B} \subset \mathscr{A}$. On the other hand, from (2.2), we have that, for any $x_{0} \in \mathscr{A}$, there exist $\left\{x_{n}\right\}_{n=1}^{\infty} \subset B_{0}$ and $\left\{t_{n}\right\}_{n=1}^{\infty} \subset \mathbb{R}^{+}$which satisfies $t_{n} \rightarrow \infty$ as $n \rightarrow \infty$ such that

$$
\left\|S\left(t_{n}\right) x_{n}-x_{0}\right\|_{Z} \rightarrow 0 \quad \text { as } n \rightarrow \infty
$$

The semigroup $\{S(t)\}_{t \geq 0}$ has an $(X, X)$-global attractor $\mathscr{B}$, consequently $\left\{S\left(t_{n}\right) x_{n}\right\}_{n=1}^{\infty}$ is precompact in $X$, then there exist a subsequence $\left\{S\left(t_{n_{k}}\right) x_{n_{k}}\right\}_{k=1}^{\infty}$ and $y_{0} \in \mathscr{B}$ such that

$$
\left\|S\left(t_{n_{k}}\right) x_{n_{k}}-y_{0}\right\|_{X} \rightarrow 0
$$

By Lemma 2.6, $x_{0}=y_{0}$, which implies that $x_{0} \in \mathscr{B}$. Noticing that $x_{0}$ is arbitrary, it immediately follows that $\mathscr{A} \subset \mathscr{B}$. The proof is complete.

Remark 2.10 By Lemma 2.6, we have that the $(X, X)$-global attractor coincides with the $(X, Y)$-global attractor.

Lemma 2.11 ([17, 19]) Let $X \subset \subset H \subset Y$ be Banach spaces with $X$ reflexive. Suppose that $u_{n}$ is a sequence that is uniformly bounded in $L^{2}(0, T ; X)$ and $d u_{n} / d t$ is uniformly bounded in $L^{p}(0, T ; Y)$ for some $p>1$. Then there is a subsequence of $u_{n}$ that converges strongly in $L^{2}(0, T ; H)$.

According to Theorem 2.9, the key to proving the existence of $\left(\mathcal{L}_{2}, \mathcal{L}_{p}\right)$-global attractors is to verify that semigroup $\{S(t)\}_{t \geq 0}$ is $\left(\mathcal{L}_{2}, \mathcal{L}_{p}\right)$-asymptotically compact. Next, our main purpose is to establish a criterion to verify the asymptotic compactness of semigroups in these bi-spaces.

Lemma 2.12 ([26]) Let $B \subset L^{2}(\Omega) \cap L^{p}(\Omega)(p \geq 2)$ be any bounded subset of $L^{2}(\Omega)$ and $L^{p}(\Omega)$. Then, for any $\varepsilon>0, B$ has a finite $\varepsilon$-net in $L^{p}(\Omega)$ if there exists a positive constant $M=M(\varepsilon)$, which depends on $\varepsilon$, such that

(1) $B$ has a finite $(3 M)^{(2-p) / 2}(\varepsilon / 2)^{p / 2}$-net in $L^{2}(\Omega)$;

(2) $\left(\int_{\Omega(|u| \geq M)}|u|^{p}\right)^{1 / p}<2^{-(2 p+2) / p} \varepsilon$ for any $u \in B$.

Lemma 2.13 ([26]) Let $\{S(t)\}_{t \geq 0}$ be a semigroup on $L^{p}(\Omega)(p \geq 1)$, and suppose that $\{S(t)\}_{t \geq 0}$ has a bounded $\left(L^{2}(\Omega), L^{p}(\Omega)\right)$-absorbing set in $L^{p}(\Omega)$. Then, for any $\varepsilon>0$ and any bounded subset $B \subset L^{p}(\Omega)$, there exist positive constants $T=T(B, \varepsilon)$ and $M=M(\varepsilon)$ such that

$$
m\left(\Omega\left(\left|S(t) u_{0}\right| \geq M\right)\right) \leq \varepsilon \quad \text { for any } u_{0} \in B, t \geq T
$$

Theorem 2.14 Let $\{S(t)\}_{t \geq 0}$ be a continuous semigroup on $\mathcal{L}_{2}$ and a semigroup on $\mathcal{L}_{p}$, where $2 \leq p<\infty$. Suppose that $\{S(t)\}_{t \geq 0}$ is $\left(\mathcal{L}_{2}, \mathcal{L}_{2}\right)$-asymptotically compact, then $\{S(t)\}_{t \geq 0}$ has an $\left(\mathcal{L}_{2}, \mathcal{L}_{p}\right)$-global attractor provided that the following conditions hold: 
(1) $\{S(t)\}_{t \geq 0}$ has an $\left(\mathcal{L}_{2}, \mathcal{L}_{p}\right)$-bounded absorbing set $B_{0}\left(\subset \mathcal{L}_{p}\right)$;

(2) for any $\varepsilon>0$ and bounded (with respect to $\|\cdot\|_{\mathcal{L}_{2}}$ ) subset $B$, there exist positive constants $M=M(\varepsilon, B)$ and $T=T(B, \varepsilon)$ such that

$$
\int_{\Omega\left(\left|\Pi_{1} S(t) z_{0}\right| \geq M\right)}\left|\Pi_{1} S(t) z_{0}\right|^{p}<\varepsilon \quad \text { for any } z_{0} \in B, t \geq T,
$$

where $\Pi_{1}$ is the projector from $X \times Y$ to $X$.

Proof By Lemma 2.9, we only need to verify that $\{S(t)\}_{t \geq 0}$ is $\left(\mathcal{L}_{2}, \mathcal{L}_{p}\right)$-asymptotically compact. Combining this embedding $L^{p}(\Omega) \hookrightarrow L^{2}(\Omega)$ is continuous with the definitions of $\mathcal{L}_{2}$ and $\mathcal{L}_{p}$, we have that the semigroup $\{S(t)\}_{t \geq 0}$ has an $\left(\mathcal{L}_{2}, \mathcal{L}_{2}\right)$-bounded absorbing set $B_{2}$. Denote $B_{p}=B_{0} \cap B_{2}$, then it is sufficient to prove that:

For any $t_{n}>0, x_{n} \in B_{p}, t_{n} \rightarrow \infty($ as $n \rightarrow \infty),\left\{S\left(t_{n}\right) x_{n}\right\}_{n=1}^{\infty}$ is precompact in $\mathcal{L}_{p}$, which is equivalent to proving that for any $\varepsilon>0,\left\{\Pi_{1} S\left(t_{n}\right) x_{n}\right\}_{n=1}^{\infty}$ has a finite $\varepsilon$-net in $L^{p}(\Omega)$. It is that the semigroup $\{S(t)\}_{t \geq 0}$ has an $\left(\mathcal{L}_{2}, \mathcal{L}_{2}\right)$-global attractor $\mathscr{A}_{0}$ and for any $t_{n}>0, x_{n} \in B_{p}, t_{n} \rightarrow$ $\infty($ as $n \rightarrow \infty),\left\{\Pi_{2} S\left(t_{n}\right) x_{n}\right\}_{n=1}^{\infty}$ is precompact in $L_{\mu}^{2}(\mathbb{R}, V)\left(\Pi_{2}\right.$ is the projector from $X \times Y$ to $Y$ ). Furthermore, $\mathcal{L}_{p}=L^{p}(\Omega) \times L_{\mu}^{2}(\mathbb{R}, V)$, so we only need to verify that $\left\{\Pi_{1} S\left(t_{n}\right) x_{n}\right\}_{n=1}^{\infty}$ is precompact in $L^{p}(\Omega)$.

In fact, from the assumption that $\{S(t)\}_{t \geq 0}$ has an $\left(\mathcal{L}_{2}, \mathcal{L}_{2}\right)$-global attractor $\mathscr{A}_{0}$, we know that there exists $T_{1}$, which only depends on $\varepsilon$ and $M$, such that $\left\{\Pi_{1} S\left(t_{n}\right) x_{n} \mid t_{n} \geq T_{1}\right\}$ has a finite $(3 M)^{(2-p) / 2}(\varepsilon / 2)^{p / 2}$-net in $L^{2}(\Omega)$. Taking $T_{0}=\max \left\{T, T_{1}\right\}$, from Lemma 2.11, we know that $\left\{\Pi_{1} S\left(t_{n}\right) x_{n} \mid t_{n} \geq T_{0}\right\}$ has a finite $\varepsilon$-net in $L^{p}(\Omega)$. Since $t_{n} \rightarrow \infty$, we obtain that $\left\{\Pi_{1} S\left(t_{n}\right) x_{n}\right\}_{n=1}^{\infty}$ has a finite $\varepsilon$-net in $L^{p}(\Omega)$, too. Then, from the arbitrariness of $\varepsilon$, we get that $\left\{\Pi_{1} S\left(t_{n}\right) x_{n}\right\}_{n=1}^{\infty}$ is precompact in $L^{p}(\Omega)$.

\section{Global attractors in $\mathcal{L}_{p}$}

Throughout the paper, we will assume conditions (1.3)-(1.6), (1.11), and $g \in L^{2}(\Omega)$. By [10] (see also [9]), the following holds.

Lemma 3.1 For any $T>0$ and every $z_{0} \in \mathcal{L}_{2}, E q$. (1.13) with initial-boundary conditions (1.14) admits a unique weak solution

$$
\begin{aligned}
& u \in L^{\infty}(0, T ; H) \cap L^{2}(0, T ; V) \cap L^{p}\left(0, T ; L^{p}(\Omega)\right), \\
& \eta^{t} \in L^{\infty}\left(0, T ; L_{\mu}^{2}\left(\mathbb{R}^{+}, V\right)\right)
\end{aligned}
$$

such that

$$
z_{t}=\mathcal{Q} z+\mathcal{G}(z),\left.\quad z\right|_{t=0}=0
$$

in the weak sense.

Furthermore,

$$
z \in C\left([0, T], \mathcal{L}_{2}\right),
$$

and the mapping

$$
z_{0} \mapsto z(t) \in C\left(\mathcal{L}_{2}, \mathcal{L}_{2}\right), \quad \forall t \in[0, T]
$$


By Lemma 3.1, we can define a semigroup $\{S(t)\}_{t \geq 0}$ in $\mathcal{L}_{2}$ as follows:

$$
\begin{aligned}
& S(t): \mathbb{R}^{+} \times \mathcal{L}_{2} \rightarrow \mathcal{L}_{2}, \\
& z_{0} \rightarrow z(t)=S(t) z_{0},
\end{aligned}
$$

and $\{S(t)\}_{t \geq 0}$ is a strongly continuous semigroup on the phase space $\mathcal{L}_{2}$.

We now deal with the dissipative feature of the semigroup $\{S(t)\}_{t \geq 0}$. Namely, we show that the trajectories originating from any given bounded set $B \subset \mathcal{L}_{2}$ eventually, uniformly in time, into a bounded absorbing set $B_{0} \subset \mathcal{L}_{p}$. For further use, let us write down explicitly the bounded absorbing set $E_{0} \subset \mathcal{L}_{2}$ of the semigroup $\{S(t)\}_{t \geq 0}$.

Lemma 3.2 For any $\mathrm{R} \geq 0$ given, there exist constants $\mathcal{R}_{0}>0$ and $t_{0}=t_{0}(\mathrm{R})$ such that, whenever

$$
\left\|z_{0}\right\|_{\mathcal{L}_{2}} \leq \mathrm{R}
$$

it follows that

$$
\left\|S(t) z_{0}\right\|_{\mathcal{L}_{2}} \leq \mathcal{R}_{0}, \quad \forall t \geq t_{0}
$$

Consequently, the

$$
E_{0}=\left\{z \in \mathcal{L}_{2}:\|z\|_{\mathcal{L}_{2}} \leq \mathcal{R}_{0}\right\}
$$

is an $\left(\mathcal{L}_{2}, \mathcal{L}_{2}\right)$-bounded absorbing set for the semigroup $\{S(t)\}_{t \geq 0}$, that is, for any bounded set $B \subset \mathcal{L}_{2}$, there is $t_{0}=t_{0}(B)$ such that $S(t) B \subset E_{0}$ for every $t \geq t_{0}$.

Proof Multiplying the first equation of (1.13) by $u$ and then integrating over $\Omega$, we get

$$
\frac{1}{2} \frac{d}{d t}|u|_{2}^{2}+\|u\|_{0}^{2}+\int_{0}^{+\infty} \mu(s)\left\langle-\Delta \eta^{t}(s), u(t)\right\rangle d s+\langle f(u), u\rangle=\langle g, u\rangle
$$

Using (1.7) and transforming the integral term in (3.2), we have

$$
\int_{0}^{+\infty} \mu(s)\left\langle-\Delta \eta^{t}(s), u(t)\right\rangle d s=\frac{1}{2} \frac{d}{d t}\left\|\eta^{t}\right\|_{\mu, 0}^{2}-\frac{1}{2} \int_{0}^{+\infty} \mu^{\prime}(s)\left\|\eta^{t}(s)\right\|_{0}^{2} d s .
$$

From (1.6), it follows that

$$
-\frac{1}{2} \int_{0}^{+\infty} \mu^{\prime}(s)\left\|\eta^{t}(s)\right\|_{0}^{2} d s \geq \frac{\delta}{2} \int_{0}^{+\infty} \mu(s)\left\|\eta^{t}(s)\right\|_{0}^{2} d s=\frac{\delta}{2}\left\|\eta^{t}\right\|_{\mu, 0}^{2} .
$$

From hypothesis (1.2) and Hölder's inequality, we obtain

$$
\langle f(u), u\rangle \geq \alpha_{1}|u|_{p}^{p}-\beta_{1}|\Omega|
$$

and

$$
\langle g, u\rangle \leq \frac{1}{2 \lambda_{1}}|g|_{2}^{2}+\frac{\lambda_{1}}{2}|u|_{2}^{2}
$$


Bringing (3.3)-(3.6) into (3.2) and combining with Poincaré's inequality, we have

$$
\frac{d}{d t}\left(|u|_{2}^{2}+\left\|\eta^{t}\right\|_{\mu, 0}^{2}\right)+\lambda_{1}|u|_{2}^{2}+\delta\left\|\eta^{t}\right\|_{\mu, 0}^{2}+2 \alpha_{1}|u|_{p}^{p} \leq \frac{1}{\lambda_{1}}|g|_{2}^{2}+2 \beta_{1}|\Omega|
$$

where $|\Omega|$ is the Lebesgue measure of $\Omega$. Taking

$$
\gamma_{1}=\min \left\{\lambda_{1}, \delta\right\}
$$

then

$$
\frac{d}{d t}\left(|u|_{2}^{2}+\left\|\eta^{t}\right\|_{\mu, 0}^{2}\right)+\gamma_{1}\left(|u|_{2}^{2}+\left\|\eta^{t}\right\|_{\mu, 0}^{2}\right) \leq \frac{1}{\lambda_{1}}|g|_{2}^{2}+2 \beta_{1}|\Omega|
$$

Applying Gronwall's lemma, we obtain

$$
|u(t)|_{2}^{2}+\left\|\eta^{t}(s)\right\|_{\mu, 0}^{2} \leq\left(\left|u_{0}(x)\right|_{2}^{2}+\left\|\eta^{0}(s)\right\|_{\mu, 0}^{2}\right) e^{-\gamma_{1} t}+\frac{1}{\gamma_{1}}\left(\frac{1}{\lambda_{1}}|g|_{2}^{2}+2 \beta_{1}|\Omega|\right) .
$$

Therefore

$$
\|z(t)\|_{\mathcal{L}_{2}}^{2} \leq\left\|z_{0}\right\|_{\mathcal{L}_{2}}^{2} e^{-\gamma_{1} t}+\frac{1}{\gamma_{1}}\left(\frac{1}{\lambda_{1}}|g|_{2}^{2}+2 \beta_{1}|\Omega|\right) .
$$

Making $\mathcal{R}_{0}^{2}=\frac{2}{\gamma_{1}}\left(\frac{1}{\lambda_{1}}|g|_{2}^{2}+2 \beta_{1}|\Omega|\right)$, when $t \geq t_{0}=\frac{1}{\gamma_{1}} \ln \frac{\gamma_{1} \lambda_{1} \mathrm{R}^{2}}{|g|_{2}^{2}+2 \lambda_{1} \beta_{1}|\Omega|}$, we obtain

$$
\|z(t)\|_{\mathcal{L}_{2}} \leq \mathcal{R}_{0}
$$

Lemma 3.3 There exists a constant $\mathcal{R}_{1}>0$ for given any $\mathrm{R} \geq 0$ such that, whenever $\left\|z_{0}\right\|_{\mathcal{L}_{2}} \leq \mathrm{R}$, the corresponding solution $z(t)=\left(u(t), \eta^{t}\right)$ fulfills

$$
\int_{t}^{t+1}\|z(s)\|_{\mathcal{L}_{p}}^{2} d s=\int_{t}^{t+1}\left(|u(s)|_{p}^{p}+\left\|\eta^{s}\right\|_{\mu, 0}^{2}\right) d s \leq \mathcal{R}_{1}
$$

and

$$
\int_{t}^{t+1}\|z(s)\|_{\mathcal{M}_{1}}^{2} d s=\int_{t}^{t+1}\left(\|u(s)\|_{0}^{2}+\left\|\eta^{s}\right\|_{\mu, 0}^{2}\right) d s \leq \mathcal{R}_{1}
$$

for all $t \geq t_{0}$ hold.

Proof From (3.2)-(3.6), we obtain

$$
\frac{d}{d t}\left(|u|_{2}^{2}+\left\|\eta^{t}\right\|_{\mu, 0}^{2}\right)+\|u\|_{0}^{2}+\delta\left\|\eta^{t}\right\|_{\mu, 0}^{2}+2 \alpha_{1}|u|_{p}^{p} \leq \frac{1}{\lambda_{1}}|g|_{2}^{2}+2 \beta_{1}|\Omega| .
$$

We integrate (3.9) about $t$ from $t$ to $t+1$ and use Lemma 3.2, then we have

$$
\int_{t}^{t+1}\|u(s)\|_{0}^{2} d s+\delta \int_{t}^{t+1}\left\|\eta^{s}\right\|_{\mu, 0}^{2} d s+2 \alpha_{1} \int_{t}^{t+1}|u(s)|_{p}^{p} d s
$$




$$
\begin{aligned}
& \leq \frac{1}{\lambda_{1}}|g|_{2}^{2}+2 \beta_{1}|\Omega|+|u(t)|_{2}^{2}+\left\|\eta^{t}(s)\right\|_{\mu, 0}^{2} \\
& \leq \frac{1}{\lambda_{1}}|g|_{2}^{2}+2 \beta_{1}|\Omega|+\|z(t)\|_{\mathcal{L}_{2}}^{2} .
\end{aligned}
$$

If we make $\mathcal{R}_{1}=\frac{1}{\min \left\{1, \delta, 2 \alpha_{1}\right\}}\left(\frac{1}{\lambda_{1}}|g|_{2}^{2}+2 \beta_{1}|\Omega|+\mathcal{R}_{0}^{2}\right)$, then when $t \geq t_{0}$, we obtain

$$
\int_{t}^{t+1}\|z(s)\|_{\mathcal{L}_{p}}^{2} d s=\int_{t}^{t+1}\left(|u(s)|_{p}^{p}+\left\|\eta^{s}\right\|_{\mu, 0}^{2}\right) d s \leq \mathcal{R}_{1}
$$

and

$$
\int_{t}^{t+1}\|z(s)\|_{\mathcal{M}_{1}}^{2} d s=\int_{t}^{t+1}\left(\|u(s)\|_{0}^{2}+\left\|\eta^{s}\right\|_{\mu, 0}^{2}\right) d s \leq \mathcal{R}_{1} .
$$

Corollary 3.4 Given any $\mathrm{R} \geq 0$, there exist constants $\mathcal{K}=\mathcal{K}(\mathrm{R})>0$ such that, whenever $\left\|z_{0}\right\|_{\mathcal{M}_{0}} \leq \mathrm{R}$, the corresponding solution $z(t)=\left(u(t), \eta^{t}\right)$ fulfills

$$
\int_{t}^{t+1}\left(|u(s)|_{p}^{p}+\|u(s)\|_{0}^{2}+\left\|\eta^{s}\right\|_{\mu, 0}^{2}\right) d s \leq \mathcal{K}
$$

for all $t \geq 0$ holds.

Proof By (3.8), we find the uniform estimate

$$
\|z(t)\|_{\mathcal{L}_{2}}^{2} \leq\left\|z_{0}\right\|_{\mathcal{L}_{2}}^{2}+\frac{1}{\gamma_{1}}\left(\frac{1}{\lambda_{1}}|g|_{2}^{2}+2 \beta_{1}|\Omega|\right), \quad \forall t \geq 0
$$

The thesis then follows from (3.10).

Lemma 3.5 The semigroup $\{S(t)\}_{t \geq 0}$ possesses an $\left(\mathcal{L}_{2}, \mathcal{L}_{p}\right)$-bounded absorbing set, that is, there are positive constants $\rho_{0}, C_{\rho_{0}}$ such that, for any bounded subset $B \subset \mathcal{L}_{2}$ and given any $\mathrm{R} \geq 0$, there exists $t_{1}\left(=t_{1}(\mathrm{R})\right)$ such that, whenever

$$
\left\|z_{0}\right\|_{\mathcal{L}_{2}} \leq \mathrm{R}
$$

it follows that

$$
\left\|S(t) z_{0}\right\|_{\mathcal{L}_{p}}^{2} \leq \rho_{0}, \quad\left\|S(t) z_{0}\right\|_{\mathcal{M}_{1}}^{2} \leq \rho_{0}
$$

and

$$
\int_{t}^{t+1}\left|u_{t}(s)\right|_{2}^{2} d s \leq C_{\rho_{0}}
$$

hold for any $t \geq t_{1}$.

Proof Multiplying the first equation of (1.13) by $u_{t}$ and then integrating over $\Omega$, we get

$$
\left|u_{t}\right|_{2}^{2}+\frac{d}{d t}\left(\|u\|_{0}^{2}+\left\langle\eta^{t}(s), u\right\rangle_{\mu, 0}+\int_{\Omega} F(u)-\int_{\Omega} g u\right)=\int_{0}^{+\infty} \mu(s)\left\langle\nabla \eta_{t}^{t}(s), \nabla u\right\rangle d s .
$$


Combining with (1.8) and (1.10), we have

$$
\begin{aligned}
& \int_{0}^{+\infty} \mu(s)\left\langle\nabla \eta_{t}^{t}(s), \nabla u\right\rangle d s \\
& =\int_{0}^{+\infty} \mu(s)\langle\nabla u, \nabla u\rangle d s-\int_{0}^{+\infty} \mu(s)\left\langle\nabla \eta_{s}^{t}(s), \nabla u\right\rangle d s \\
& \leq \frac{5 m_{0}}{4}\|u(t)\|_{0}^{2}+\int_{0}^{+\infty} \mu(s)\left\|\eta_{s}^{t}(s)\right\|_{0}^{2} d s, \\
& \int_{0}^{+\infty} \mu(s)\left\|\eta_{s}^{t}(s)\right\|_{0}^{2} d s \\
& =\int_{0}^{+\infty} \mu(s)\|u(t-s)\|_{0}^{2} d s \\
& =\int_{0}^{t} \mu(t-s)\|u(s)\|_{0}^{2} d s+\int_{0}^{+\infty} \mu(t+s)\|u(-s)\|_{0}^{2} d s \\
& \leq e^{-\delta\left(t-t_{0}\right)} e^{-\delta t_{0}} \int_{0}^{t_{0}} e^{-\delta s}\|u(s)\|_{0}^{2} d s+e^{-\delta t} \int_{t_{0}}^{t} e^{\delta s}\|u(s)\|_{0}^{2} d s+e^{-\delta t} \Re \\
& \leq C e^{-\delta t}\left(e^{\delta t_{0}} \mathcal{K}+\mathfrak{R}\right)+C \mathcal{R}_{1},
\end{aligned}
$$

where $\mathfrak{R}$ from (1.11). By (3.12), we get

$$
\begin{aligned}
& \frac{d}{d t}\left(\|u\|_{0}^{2}+\left\langle\eta^{t}(s), u\right\rangle_{\mu, 0}+\int_{\Omega} F(u)-\int_{\Omega} g u\right) \\
& \quad \leq \frac{5 m_{0}}{4}\|z(t)\|_{\mathcal{M}_{1}}^{2}+C e^{-\delta t}\left(e^{\delta t_{0}} \mathcal{K}+\Re\right)+C \mathcal{R}_{1} .
\end{aligned}
$$

\section{Setting}

$$
E(t)=\|u\|_{0}^{2}+\left\langle\eta^{t}(s), u\right\rangle_{\mu, 0}+\int_{\Omega} F(u)-\int_{\Omega} g u,
$$

then from (1.4) we get

$$
\begin{aligned}
E(t) & \leq 2\|u\|_{0}^{2}+\frac{m_{0}}{2}\left\|\eta^{t}\right\|_{\mu, 0}^{2}+\tilde{\alpha}_{2}|u|_{p}^{p}+\tilde{\beta}_{2}|\Omega|+\frac{1}{2 \lambda_{1}}|g|_{2}^{2} \\
& \leq C\left(1+\|z(t)\|_{\mathcal{M}_{1}}^{2}+\|z(t)\|_{\mathcal{L}_{p}}^{2}\right)
\end{aligned}
$$

and

$$
\begin{aligned}
E(t) & \geq \frac{1}{2}\|u\|_{0}^{2}-m_{0}\left\|\eta^{t}\right\|_{\mu, 0}^{2}+\tilde{\alpha}_{1}|u|_{p}^{p}-\tilde{\beta}_{1}|\Omega|-\frac{1}{\lambda_{1}}|g|_{2}^{2} \\
& \geq C\left(\|z(t)\|_{\mathcal{M}_{1}}^{2}+\|z(t)\|_{\mathcal{L}_{p}}^{2}\right)-C\left(1+\|z(t)\|_{\mathcal{L}_{2}}^{2}\right) .
\end{aligned}
$$

Integrating (3.14) about $t$ from $s(s \geq t)$ to $t+1$, we obtain

$$
E(t+1) \leq C \int_{t}^{t+1}\|z(s)\|_{\mathcal{M}_{1}}^{2} d s+\frac{C}{\delta} e^{-\delta s}\left(e^{\delta t_{0}} \mathcal{K}+\Re\right)+C \mathcal{R}_{1}+E(s)
$$


Integrating (3.17) about $s$ from $t$ to $t+1$, we get

$$
E(t+1) \leq C \int_{t}^{t+1}\|z(s)\|_{\mathcal{M}_{1}}^{2} d s+\frac{C}{\delta^{2}} e^{-\delta t}\left(e^{\delta t_{0}} \mathcal{K}+\Re\right)+C \mathcal{R}_{1}+\int_{t}^{t+1} E(s) d s .
$$

Combining with (3.15) and (3.16), it follows that

$$
\begin{aligned}
\| z(t+1) & \left\|_{\mathcal{M}_{1}}^{2}+\right\| z(t+1) \|_{\mathcal{L}_{p}}^{2} \\
\leq & C \int_{t}^{t+1}\left(\|z(s)\|_{\mathcal{M}_{1}}^{2}+\|z(s)\|_{\mathcal{L}_{p}}^{2}\right) d s+C\left(1+\|z(t+1)\|_{\mathcal{L}_{p}}^{2}\right) \\
& +\frac{C}{\delta^{2}} e^{-\delta t}\left(e^{\delta t_{0}} \mathcal{K}+\Re\right)+C \mathcal{R}_{1} .
\end{aligned}
$$

By Lemma 3.2 and Lemma 3.3, there is a positive constant $\rho_{0}$ such that

$$
\|z(t)\|_{\mathcal{M}_{1}}^{2}+|u(t)|_{p}^{p} \leq \rho_{0}
$$

for all $t \geq t_{1}=t_{0}+\frac{1}{\delta} \ln \left(\frac{e^{\delta t_{0}} \mathcal{K}+\Re}{\delta^{2} \mathcal{R}_{1}}\right)$ holds.

By (3.11) and (3.12), we have

$$
\int_{t}^{t+1}\left|u_{t}(s)\right|_{2}^{2} d s+E(t+1) \leq C_{0} \int_{t}^{t+1}\|z(s)\|_{\mathcal{M}_{1}}^{2} d s+E(t) .
$$

Associating with (3.15), (3.16), and (3.20), we obtain that there exists a positive constant $C_{\rho_{0}}$ such that

$$
\int_{t}^{t+1}\left|u_{t}(s)\right|_{2}^{2} d s \leq C_{\rho_{0}}
$$

for all $t \geq t_{1}$ holds.

Remark 3.6 By Lemma 3.5, we can obtain that the semigroup $\{S(t)\}_{t \geq 0}$ corresponding to Eq. (1.13) possesses an $\left(\mathcal{L}_{2}, \mathcal{L}_{p}\right)$-bounded absorbing set $B_{0}$ :

$$
B_{0}=\left\{\left(u, \eta^{t}\right) \in H_{0}^{1}(\Omega) \cap L^{p}(\Omega) \times L_{\mu}^{2}\left(\mathbb{R}^{+}, H_{0}^{1}(\Omega)\right):\|u\|_{0}^{2}+\left\|\eta^{t}\right\|_{\mu, 0}^{2}+|u|_{p}^{p} \leq \rho_{0}\right\} .
$$

It is obvious that $B_{0}$ is also an $\left(\mathcal{L}_{2}, \mathcal{M}_{1}\right)$-bounded absorbing set of the semigroup $\{S(t)\}_{t \geq 0}$.

Lemma 3.7 There exist constants $\mathcal{K}_{1}, \mathcal{K}_{2}>0$, for given any $z_{0} \in B_{0}$ (from 3.23), the corresponding solution $z(t)=\left(u(t), \eta^{t}\right)$ fulfills

$$
\left|u_{t}(t)\right|_{2}^{2} \leq \mathcal{K}_{1}
$$

and

$$
\int_{t}^{t+1}\left\|u_{t}(s)\right\|_{0}^{2} d s \leq \mathcal{K}_{2}
$$

for all $t>0$ hold. 
Proof Differentiating about $t$ for Eq. (1.13), and using (1.8), (1.10), we obtain

$$
\left\{\begin{array}{l}
u_{t t}-\Delta u_{t}-\int_{0}^{+\infty} \mu(s) \Delta \eta_{t}^{t}(s) d s+f^{\prime}(u) u_{t}=0 \\
u_{t}=\eta_{t t}^{t}+\eta_{t s}^{t}
\end{array}\right.
$$

Multiplying the first equation of (3.26) by $u_{t}$ and then integrating over $\Omega$, it follows that

$$
\frac{d}{d t}\left(\left|u_{t}\right|_{2}^{2}+\left\|\eta_{t}^{t}\right\|_{\mu, 0}^{2}\right)+\left\|u_{t}\right\|_{0}^{2}+\delta\left\|\eta_{t}^{t}\right\|_{\mu, 0}^{2} \leq 2 l\left|u_{t}\right|_{2}^{2}
$$

Now, we investigate the estimate of $\left\|\eta_{t}^{t}\right\|_{\mu, 0}^{2}$ :

$$
\begin{aligned}
\left\|\eta_{t}^{t}\right\|_{\mu, 0}^{2} & =\int_{0}^{\infty} \mu(s)\|u(t)-u(t-s)\|_{0}^{2} d s \\
& \leq 2 m_{0}\|u(t)\|_{0}^{2}+2 \int_{0}^{\infty} \mu(s)\|u(t-s)\|_{0}^{2} d s .
\end{aligned}
$$

And

$$
\begin{aligned}
\int_{0}^{\infty} \mu(s)\|u(t-s)\|_{0}^{2} d s & =\int_{-t}^{\infty} \mu(t+s)\|u(-s)\|_{0}^{2} d s \\
& =\int_{-t}^{0} \mu(t+s)\|u(-s)\|_{0}^{2} d s+\int_{0}^{\infty} \mu(t+s)\|u(-s)\|_{0}^{2} d s \\
& =\int_{0}^{t} \mu(t-s)\|u(s)\|_{0}^{2} d s+\int_{0}^{\infty} \mu(t+s)\|u(-s)\|_{0}^{2} d s \\
& \leq \frac{1}{\delta} \rho_{0}+e^{-\delta t} \Re .
\end{aligned}
$$

Hence, for any $t \geq 0$, we get

$$
\left\|\eta_{t}^{t}\right\|_{\mu, 0}^{2} \leq C \rho_{0}+2 e^{-\delta t} \mathfrak{R}
$$

and

$$
\eta_{t}^{t} \in L_{\mu}^{2}\left(\mathbb{R}, H_{0}^{1}(\Omega)\right)
$$

Integrating (3.27) about $t$ from $s$ to $t(0<s \leq t \leq 1)$, we obtain

$$
\left|u_{t}(t)\right|_{2}^{2}+\left\|\eta_{t}^{t}\right\|_{\mu, 0}^{2} \leq 2 l \int_{0}^{1}\left|u_{t}(s)\right|_{2}^{2} d s+\left|u_{t}(s)\right|_{2}^{2}+\left\|\eta_{t}^{s}\right\|_{\mu, 0}^{2}
$$

Then integrating (3.29) about $s$ from 0 to 1 , for any $0<t \leq 1$, it follows that

$$
\left|u_{t}(t)\right|_{2}^{2} \leq(2 l+1) \int_{0}^{1}\left|u_{t}(s)\right|_{2}^{2} d s+C \rho_{0}+2 \Re
$$

By Lemma 3.5, for all $0<t \leq 1$, we get

$$
\left|u_{t}(t)\right|_{2}^{2} \leq(2 l+1) C_{\rho_{0}}+C \rho_{0}+2 \Re
$$


Let $s \in(0,1]$, using Gronwall's lemma to (3.27) and combining with (3.30), for any $t \geq s$, we have

$$
\begin{aligned}
\left|u_{t}(t)\right|_{2}^{2}+\left\|\eta_{t}^{t}\right\|_{\mu, 0}^{2} & \leq\left(\left|u_{t}(s)\right|_{2}^{2}+\left\|\eta_{t}^{s}\right\|_{\mu, 0}^{2}\right) e^{-\delta(t-s)}+(2 l+\delta) e^{-\delta t} \int_{0}^{t} e^{\delta s}\left|u_{t}(s)\right|_{2}^{2} d s \\
& \leq(2 l+\delta) C_{\rho_{0}}+C \rho_{0}+2 \Re+\frac{(2 l+\delta) e}{1-e^{-\delta}}
\end{aligned}
$$

Setting

$$
\mathcal{K}_{1}=(2 l+1) C_{\rho_{0}}+C \rho_{0}+2 \Re+\frac{(2 l+\delta) e}{1-e^{-\delta}},
$$

then for any $t>0$, it follows that

$$
\left|u_{t}(t)\right|_{2}^{2} \leq \mathcal{K}_{1}
$$

For any $t>0$, we integrate (3.27) about $t$ on $[t, t+1]$, then

$$
\int_{t}^{t+1}\left\|u_{t}(s)\right\|_{0}^{2} d s \leq 2 l \int_{t}^{t+1}\left|u_{t}(s)\right|_{2}^{2} d s+\left|u_{t}(t)\right|_{2}^{2}+\left\|\eta_{t}^{t}\right\|_{\mu, 0}^{2} .
$$

Using Lemma 3.5 and (3.32) and letting $\mathcal{K}_{2}=2 l C_{\rho_{0}}+\mathcal{K}_{1}$, we obtain

$$
\int_{t}^{t+1}\left\|u_{t}(s)\right\|_{0}^{2} d s \leq \mathcal{K}_{2}
$$

In order to prove the existence of an $\left(\mathcal{L}_{2}, \mathcal{L}_{p}\right)$-global attractor for $\{S(t)\}_{t \geq 0}$, and for further purposes, we first have to verify that the semigroup $\{S(t)\}_{t \geq 0}$ is asymptotically compact on $\mathcal{L}_{2}$.

Lemma 3.8 The semigroup $\{S(t)\}_{t \geq 0}$ associated with problem (1.13) with initial and boundary values $(1.14)$ is $\left(\mathcal{L}_{2}, \mathcal{L}_{2}\right)$-asymptotically compact.

Proof Let $z^{1}(t)=\left(u^{1}(t), \eta_{1}^{t}\right), z^{2}(t)=\left(u^{2}, \eta_{2}^{t}\right)$ be two solutions of (1.13) corresponding to the initial data $z_{0}^{1}=\left(u_{0}^{1}, \eta_{1}^{0}\right), z_{0}^{2}=\left(u_{0}^{2}, \eta_{2}^{0}\right)$ respectively. Set $z(t)=\left(\omega(t), \theta^{t}\right)=\left(u^{1}(t)-u^{2}(t), \eta_{1}^{t}-\right.$ $\left.\eta_{2}^{t}\right)$, then $z(t)$ satisfies the following equation:

$$
\omega_{t}-\Delta \omega-\int_{0}^{+\infty} \mu(s) \Delta \theta^{t}(s) d s+f\left(u^{1}\right)-f\left(u^{2}\right)=0,
$$

with initial-boundary conditions

$$
\left\{\begin{array}{l}
\left.\omega(x, t)\right|_{\partial \Omega}=0,\left.\quad \theta^{t}(x, s)\right|_{\partial \Omega \times \mathbb{R}^{+}}=0, \\
\omega(x, 0)=u_{0}^{1}-u_{0}^{2}, \quad \theta^{0}(x, s)=\eta_{1}^{0}-\eta_{2}^{0} .
\end{array}\right.
$$

Multiplying (3.33) by $\omega$ and then integrating in $\Omega$, we get

$$
\frac{1}{2} \frac{d}{d t}\left(|\omega|_{2}^{2}+\left\|\theta^{t}\right\|_{\mu, 0}^{2}\right)+\|\omega\|_{0}^{2}+\frac{\delta}{2}\left\|\theta^{t}\right\|_{\mu, 0}^{2} \leq l|\omega|_{2}^{2}
$$


Making $\gamma=\min \left\{2 \lambda_{1}, \delta\right\}$, by Gronwall's lemma, we obtain

$$
|\omega(T)|_{2}^{2}+\left\|\theta^{T}\right\|_{\mu, 0}^{2} \leq\left(|\omega(0)|_{2}^{2}+\left\|\theta^{0}\right\|_{\mu, 0}^{2}\right) e^{-\gamma T}+2 l \int_{0}^{T}|\omega(s)|_{2}^{2} d s
$$

For any $\varepsilon>0$, let $T=\frac{1}{\gamma} \ln \frac{|\omega(0)|_{2}^{2}+\left\|\theta^{0}\right\|_{\mu, 0}^{2}}{\varepsilon^{2}}$, then we get

$$
\left\|S(T) z^{1}-S(T) z^{2}\right\|_{\mathcal{L}_{2}} \leq \varepsilon+\phi_{T}\left(z^{1}, z^{2}\right)
$$

where

$$
\phi_{T}\left(z^{1}, z^{2}\right)=\left(2 l \int_{t_{1}}^{T}\left|u^{1}(s)-u^{2}(s)\right|_{2}^{2} d s\right)^{1 / 2} .
$$

By Lemma 3.5, using Lemma 2.11, then $\phi_{T}$ is a contractive function on $B_{0}$.

Corollary 3.9 The semigroup $\{S(t)\}_{t \geq 0}$ possesses an $\left(\mathcal{L}_{2}, \mathcal{L}_{2}\right)$-global attractor $\mathscr{A}_{0}$.

Lemma 3.10 The semigroup $\{S(t)\}_{t \geq 0}$ associated with problem (1.13) with initial and boundary values $(1.14)$ is $\left(\mathcal{L}_{2}, \mathcal{L}_{p}\right)$-asymptotically compact.

Proof Applying once more Theorem 2.14, Lemma 3.1, and Lemma 3.2, we only prove that, for any $\varepsilon>0$ and bounded (with respect to $\|\cdot\|_{\mathcal{L}_{2}}$ ) subset $B$, there exist positive constants $M=M(\varepsilon, B)$ and $T=T(B, \varepsilon)$ such that

$$
\int_{\Omega(|u| \geq M)}|u|^{p}<\varepsilon \quad \text { for any } z_{0} \in B, t \geq T
$$

where $u=u(t)=\Pi_{1} z(t)$.

From Lemma 3.3 and Remark 3.6, there exist positive constants $M_{1}=M_{1}(\varepsilon, B)$ and $T_{0}=$ $T_{0}(B, \varepsilon)$ such that

$$
m\left(\Omega\left(|u| \geq M_{1}\right)\right) \leq \varepsilon
$$

and

$$
\int_{\Omega\left(|u| \geq M_{1}\right)}|g|^{2} \leq \varepsilon
$$

where $m(e)$ denote the Lebesgue measure of $e \subset \Omega$ and $\left(\Omega\left(|u| \geq M_{1}\right)\right)=\{x \in \Omega:|u(x)| \geq$ $\left.M_{1}\right\}$.

Let $\left.\Omega_{1}=\Omega\left(|u| \geq M_{1}\right)\right)$ and denote

$$
\left(u-M_{1}\right)_{+}=\left\{\begin{array}{ll}
u-M_{1} & \text { as } u \geq M_{1}, \\
0 & \text { as } u<M_{1} .
\end{array} \quad\left(u+M_{1}\right)_{-}= \begin{cases}u+M_{1} & \text { as } u \leq-M_{1}, \\
0 & \text { as } u>-M_{1} .\end{cases}\right.
$$


Multiplying the first equation of $(1.13)$ by $\left(u-M_{1}\right)_{+}$and integrating over $\Omega$, we have

$$
\begin{aligned}
& \frac{1}{2} \frac{d}{d t}\left(\left|\left(u-M_{1}\right)_{+}\right|_{2}^{2}+\int_{0}^{\infty} \mu(s) \int_{\Omega_{1}}\left|\nabla \eta^{t}(s)\right|^{2} d s\right)+\int_{\Omega_{1}}|\nabla u|^{2} \\
& \quad+\frac{\delta}{2} \int_{0}^{\infty} \mu(s) \int_{\Omega_{1}}\left|\nabla \eta^{t}(s)\right|^{2} d s \\
& \int_{\Omega_{1}} f(u)\left(u-M_{1}\right)_{+}=\int_{\Omega_{1}} g\left(u-M_{1}\right)_{+} .
\end{aligned}
$$

For $M_{1}$ large enough, we get

$$
f(u)\left(u-M_{1}\right)_{+} \geq 0 .
$$

By Hölder's inequality and Cauchy's inequality, we know that

$$
\begin{aligned}
& \frac{1}{2} \frac{d}{d t}\left(\left|\left(u-M_{1}\right)_{+}\right|_{2}^{2}+\int_{0}^{\infty} \mu(s) \int_{\Omega_{1}}\left|\nabla \eta^{t}(s)\right|^{2} d s\right) \\
& \quad+C\left(\int_{\Omega_{1}}\left|\left(u-M_{1}\right)_{+}\right|^{2}+\int_{0}^{\infty} \mu(s) \int_{\Omega_{1}}\left|\nabla \eta^{t}(s)\right|^{2} d s\right) \leq C \varepsilon .
\end{aligned}
$$

Thus there exists $T_{1}>0$, for any $t \geq T_{1}$, we get

$$
\left|(u-M)_{+}\right|_{2}^{2}+\int_{0}^{\infty} \mu(s) \int_{\Omega_{1}}\left|\nabla \eta^{t}(s)\right|^{2} d s \leq C \varepsilon .
$$

Combining with (3.37), integrating (3.36) from $t$ to $t+1$, yields

$$
\int_{t}^{t+1}\left(\int_{\Omega_{1}}|\nabla u|^{2}+\int_{0}^{\infty} \mu(s) \int_{\Omega_{1}}\left|\nabla \eta^{t}(s)\right|^{2} d s+\int_{\Omega_{1}} f(u)\left(u-M_{1}\right)_{+}\right) \leq C \varepsilon .
$$

Hence, for any $t \geq T_{1}$,

$$
\int_{t}^{t+1}\left(\int_{\Omega_{2}}|\nabla u|^{2}+\int_{0}^{\infty} \mu(s) \int_{\Omega_{2}}\left|\nabla \eta^{t}(s)\right|^{2} d s+\int_{\Omega_{2}} f(u) u\right) \leq C \varepsilon,
$$

where $\left.\Omega_{2}=\Omega\left(u \geq 2 M_{1}\right)\right)$.

Furthermore, we multiply the first equation of (1.13) by $\left(u-2 M_{1}\right)_{+t}$ and integrating over $\Omega$, then we get

$$
\begin{aligned}
\frac{d}{d t}\left(\frac{1}{2} \int_{\Omega_{2}}|\nabla u|_{2}^{2}+\int_{\Omega_{2}} F(u)\right) & \leq\left\|u_{t}\right\|_{0}\left(\int_{0}^{\infty} \mu(s) \int_{\Omega_{2}}\left|\nabla \eta^{t}(s)\right|^{2} d s\right)^{1 / 2}+C \varepsilon \\
& \leq C\left(\left\|u_{t}\right\|_{0}+1\right) \varepsilon .
\end{aligned}
$$

Hence, we have

$$
\begin{aligned}
\int_{\Omega_{2}} F(u) \leq & \int_{t}^{t+1}\left(\frac{1}{2} \int_{\Omega_{2}}|\nabla u(s)|_{2}^{2}+\int_{\Omega_{2}} F(u(s))\right) d s \\
& +C\left(\left(\int_{t}^{t+1}\left\|u_{t}(s)\right\|_{0} d s\right)^{1 / 2}+1\right) \varepsilon .
\end{aligned}
$$


From (1.2), (1.4), (3.39), and Lemma 3.7, we obtain that

$$
\int_{\Omega\left(u \geq 2 M_{1}\right)}|u|^{p} \leq C \varepsilon
$$

Repeating the steps above, just replacing $\left(u-M_{1}\right)_{+},\left(u-2 M_{1}\right)_{+}$with $\left(u+M_{1}\right)_{-},\left(u+2 M_{1}\right)_{-}$ respectively, we deduce that

$$
\int_{\Omega\left(u \leq-2 M_{1}\right)}|u|^{p} \leq C \varepsilon
$$

Let $T=\max \left\{T_{0}, T_{1}\right\}$ and $M=2 M_{1}$, then we have that

$$
\int_{\Omega(|u| \geq M)}|u|^{p} \leq C \varepsilon
$$

for any $t \geq T$ holds.

It follows that $\{S(t)\}_{t \geq 0}$ is a norm-to-weak continuous semigroup on $\mathcal{L}_{p}$ with a bounded absorbing set $B_{0}$ and $\left(\mathcal{L}_{2}, \mathcal{L}_{p}\right)$-asymptotically compact. Then, obviously, by the standard method for dynamical systems, see for example $[2,5,20]$, we know that $\mathscr{A}$ is invariant, compact in $\mathcal{L}_{p}$, and attracts every bounded subset of $\mathcal{L}_{2}$ with respect to the $\mathcal{L}_{p}$-norm. Furthermore, by Remark 2.10, Lemma 3.8, and Lemma 3.10,

$$
\mathscr{A}_{0}=\mathscr{A} .
$$

\section{Acknowledgements}

The authors would like to thank the referees for their many helpful comments and suggestions.

\section{Funding}

The research is financially supported by Hunan Provincial Key Laboratory of Mathematical Modeling and Analysis in Engineering (Changsha University of Science and Technology), the Province Natural Science Foundation of Hunan (Nos. 2018JJ2416), and the National Natural Science Foundation of China (Nos. 11101053, 71471020).

\section{Availability of data and materials}

Not applicable.

\section{Competing interests}

The authors declare to have no competing interests.

\section{Authors' contributions}

The first and second authors played a major role in this work. The authors investigated the literature reviews. All authors read and approved the final manuscript.

\section{Publisher's Note}

Springer Nature remains neutral with regard to jurisdictional claims in published maps and institutional affiliations.

Received: 16 July 2019 Accepted: 1 November 2019 Published online: 11 December 2019

\section{References}

1. Aifantis, E.: On the problem of diffusion in solids. Acta Mech. 37(3-4), 265-296 (1980)

2. Babin, A.V., Vishik, M.I.: Attractors of Evolution Equations. North-Holland, Amsterdam (1992)

3. Chepyzhov, V.V., Gattib, S., Grassellic, M., Miranvilled, A., Pata, V.: Trajectory and global attractors for evolution equations with fading memory. Appl. Math. Lett. 19(1), 87-96 (2006)

4. Chepyzhov, V.V., Miranville, A.: On trajectory and global attractors for semilinear heat equations with fading memory. Indiana Univ. Math. J. 55(1), 119-167 (2006)

5. Cholewa, J.W., Dlotko, T.: Bi-spaces global attractors in abstract parabolic equations. Banach Cent. Publ. 60, 13-26 (2003) 
6. Conti, M., Gatti, S., Grasselli, M., Pata, V.: Two-dimensional reaction-diffusion equations with memory. Q. Appl. Math. $68,607-643(2010)$

7. Dafermos, C.M.: Asymptotic stability in viscoelasticity. Arch. Ration. Mech. Anal. 37(4), 297-308 (1970)

8. Gatti, S., Grasselli, M., Pata, V.: Lyapunov functionals for reaction diffusion equations with memory. Math. Methods Appl. Sci. 28(14), 1725-1735 (2005)

9. Giorgi, C., Naso, M.G., Pata, V.: Exponential stability in linear heat conduction with memory: a semigroup approach. Commun. Appl. Anal. 5(1), 121-133 (2001)

10. Giorgi, C., Pata, V., Marzocchi, A.: Asymptotic behavior of a semilinear problem in heat conduction with memory. NoDEA Nonlinear Differ. Equ. Appl. 5(3), 333-354 (1998)

11. Gurtin, M.E., Pipkin, A.: A general theory of heat conduction with finite wave speed. Arch. Ration. Mech. Anal. 31, $113-126(1968)$

12. Jackle, J.: Heat conduction and relaxation in liquids of high viscosity. Phys. Rev. A 162(3), 377-404 (1990)

13. Khanmamedov, A.K.: Global attractors for von Karman equations with nonlinear interior dissipation. J. Math. Anal. Appl. 318, 92-101 (2006)

14. Li, J., Liu, F., Feng, L., Turner, I.: A novel finite volume method for the Riesz space distributed-order diffusion equation Comput. Math. Appl. 74, 772-783 (2017)

15. Meixner, J.: On the linear theory of heat conduction. Arch. Ration. Mech. Anal. 39(2), 108-130 (1970)

16. Nunziato, J.: On heat conduction in materials with memory. Q. Appl. Math. 29, 187-204 (1971)

17. Robinson, J.C.: Infinite-Dimensional Dynamical Systems an Introduction to Dissipative Parabolic PDEs and Theory of Global Attractors. Cambridge University Press, Cambridge (2001)

18. Sun, S., Dao, D., Duan, J.: Uniform attractors for nonautonomous wave equations with nonlinear damping. SIAM J. Appl. Dyn. Syst. 6(2), 293-318 (2008)

19. Temam, T.: Infinite Dimensional Dynamical System in Mechanics and Physics, 2nd edn. Springer, New York (1997)

20. Wang, F., Wang, P., Yao, Z:: Approximate controllability of fractional partial differential equation. Adv. Differ. Equ. 2015, 367 (2015)

21. Wang, F., Yao, Z.: Approximate controllability of fractional neutral differential systems with bounded delay. Fixed Point Theory 17(2), 495-508 (2016)

22. Xie, Y., Li, Q., Huang, C., Jiang, Y.: Attractors for the semilinear reaction-diffusion equation with distribution derivatives. J. Math. Phys. 54, 092702 (2013)

23. Xie, Y., Li, Q., Zhu, K.: Attractors for nonclassical diffusion equations with arbitrary polynomial growth nonlinearity Nonlinear Anal., Real World Appl. 31, 23-37 (2016)

24. Xie, Y., Li, Y., Zeng, Y.: Uniform attractors for nonclassical diffusion equations with memory. J. Funct. Spaces 2016(3-4), $1-11(2016)$

25. Xie, Y., Zhang, J., Li, J., Luo, Q.: Attractors for reaction-diffusion equations with memory. To submit

26. Zhong, C., Yang, M., Sun, C.: The existence of global attractors for the norm-to-weak continuous semigroup and application to the nonlinear reaction-diffusion equations. J. Differ. Equ. 223(2), 367-399 (2006)

27. Zhu, K., Xie, Y., Zhou, F.: $L^{p}$-pullback attractors for non-autonomous reaction-diffusion equations with delays. Topol. Methods Nonlinear Anal. (in press). https://doi.org/10.12775/TMNA.2019.020

28. Zhu, K., Xie, Y., Zhou, F., Li, X.: Pullback attractors for non-autonomous reaction-diffusion equations in $\mathbb{R}^{n}$. J. Math. Phys. 60, $0032702(2019)$

\section{Submit your manuscript to a SpringerOpen ${ }^{\circ}$ journal and benefit from:}

- Convenient online submission

- Rigorous peer review

- Open access: articles freely available online

- High visibility within the field

- Retaining the copyright to your article

Submit your next manuscript at $\boldsymbol{~ s p r i n g e r o p e n . c o m ~}$ 